This item was submitted to Loughborough's Research Repository by the author.

Items in Figshare are protected by copyright, with all rights reserved, unless otherwise indicated.

\title{
Estimating skeletal muscle fascicle curvature from B-mode ultrasound image sequences
}

PLEASE CITE THE PUBLISHED VERSION

http://dx.doi.org/10.1109/TBME.2013.2245328

PUBLISHER

(C) IEEE

VERSION

AM (Accepted Manuscript)

\section{PUBLISHER STATEMENT}

This work is made available according to the conditions of the Creative Commons Attribution-NonCommercialNoDerivatives 4.0 International (CC BY-NC-ND 4.0) licence. Full details of this licence are available at: https://creativecommons.org/licenses/by-nc-nd/4.0/

\section{LICENCE}

CC BY-NC-ND 4.0

\section{REPOSITORY RECORD}

Darby, John, Baihua Li, Nicholas Costen, Ian Loram, and Emma Hodson-Tole. 2019. "Estimating Skeletal Muscle Fascicle Curvature from B-mode Ultrasound Image Sequences". figshare. https://hdl.handle.net/2134/20257. 


\title{
Estimating Skeletal Muscle Fascicle Curvature from B-Mode Ultrasound Image Sequences
}

\author{
John Darby, Baihua Li, Nicholas Costen, Ian Loram, Emma Hodson-Tole
}

\begin{abstract}
We address the problem of tracking in vivo muscle fascicle shape and length changes using ultrasound video sequences. Quantifying fascicle behaviour is required to improve understanding of the functional significance of a muscle's geometric properties. Ultrasound imaging provides a non-invasive means of capturing information on fascicle behaviour during dynamic movements, to date however computational approaches to assess such images are limited. Our approach to the problem is novel because we permit fascicles to take up non-linear shape configurations. We achieve this using a Bayesian tracking framework that is: i) robust, conditioning shape estimates on the entire history of image observations; and ii) flexible, enforcing only a very weak Gaussian Process shape prior that requires fascicles to be locally smooth. The method allows us to track and quantify fascicle behaviour in vivo during a range of movements, providing insight into dynamic changes in muscle geometric properties which may be linked to patterns of activation and intramuscular forces and pressures.
\end{abstract}

Index Terms-Medical imaging, image analysis, tracking, particle filtering, importance sampling, KLT, locomotion.

\section{INTRODUCTION}

$\mathbf{S}$ KELETAL muscles are highly organised structures composed of passive, elastic tissues (e.g. tendon) and of tissues which, in response to signals from the central nervous system, actively contract and produce force. The active tissues are termed muscle fibres and within the muscle they are grouped into small bundles, termed fascicles. When a muscle is activated the length and orientation of the fibres, and hence fascicles, transiently changes. Measuring these changes is of great interest to many in the biological fields of muscle mechanics and motor control and informs current understanding of the mechanical properties of skeletal muscle.

Muscle fascicles are visible within B-mode ultrasound images and this imaging modality has been used to noninvasively quantify changes in fascicle geometry during completion of different motor tasks. To date, many studies have involved manually labelling fascicle shapes in collected images [1], [2], however, the process is slow and open to human bias and error. For these reasons automated, computational approaches that make use of image processing techniques have been sought. Many of these approaches, however, make

This paper has supplementary downloadable material available at http://ieeexplore.ieee.org, provided by the authors. This includes nine multimedia WMV format movie clips, which show tracking results from the proposed algorithm. This material is $72.9 \mathrm{MB}$ in size.

All authors are at the Manchester Metropolitan University, Manchester, M1 5GD, UK. J. Darby, B. Li and N. Costen are with the School of Computing, Mathematics and Digital Technology. I. Loram and E. Hodson-Tole are with the School of Healthcare Science. E-mail: \{j.darby, b.li, n.costen, i.loram, e.tole\}@mmu.ac.uk

Manuscript received July 13, 2012. strong assumptions about the shape of the observed fascicles, in particular that they are straight [3], [4], [5], [6]. Some mathematical models [7] and experimental studies [1], [2], [8], [9] have indicated that fascicles may in fact curve. This has important implications for the accurate calculation of the mechanical behaviour of the muscle (often investigated using measures of fascicle strain and strain rate) and for understanding factors determining the mechanical stability of a muscle [7]. It is therefore desirable to develop an automated approach without strong shape constraints to permit accurate characterisation of dynamic changes in a muscle's geometric properties. To our knowledge, there are just a handful of automated approaches that are (theoretically, if not always practically) able to detect and quantify non-linear fascicle characteristics from ultrasound video sequences.

One approach, proposed by Cronin et al. [6], treats the fascicle region as a single (manually defined) patch, allowed to undergo affine warps (i.e. deformations). In the first frame of a sequence a fascicle is defined with a set of connected vertices lying within the patch. By exposing these component vertices to the recovered warps between consecutive frames, subsequent fascicle shapes are estimated. The reported work only considers length and orientation of a straight fascicle, $N=2$ vertices. The authors note, however, that it may be possible to account for curvature if fascicles are defined using $N>2$ vertices, leaving an outstanding question of whether the class of affine transformations is sufficiently rich to capture the movements of the fascicle region.

In contrast to modelling the fascicle region as a single deformable patch, other work has broken the problem into one of small [10], distinctive [11] image patches that, during tracking, are permitted to move with some level of [10] or complete [11] independence. Again, such approaches could in theory be used to track curved fascicle configurations by defining an initial fascicle with $N>2$ points and either assigning templates to vertices [10] or interpolating the effect of template movement on vertices [11], however these procedures are untested.

Namburete et al. [12] estimate instantaneous fascicle curvature measures from single images. They first estimate local orientation across a (manually defined) fascicle region with a wavelet analysis approach [4], before averaging orientations over larger image blocks, and generating fascicle trajectories from a number of random seed points with Fibre Assignment by Continuous Tracking [13], [14]. The authors do not show any individual fascicle trajectories from real ultrasound data, but by calculating curvature measures from the collection of resulting trajectories, they were able to map the local curvature in the fascicle region and demonstrate its inhomogeneous 


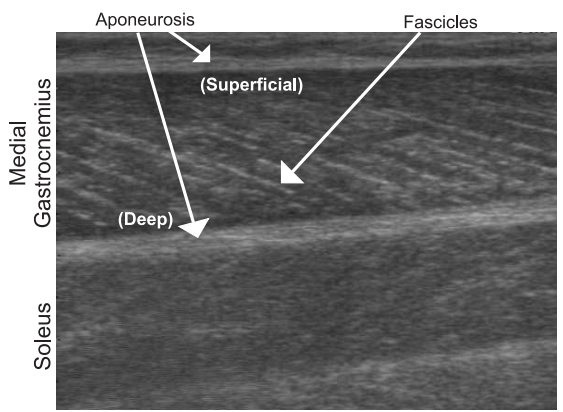

Fig. 1. Example image from the medial gastrocnemius (MG) muscle at the back of the lower leg.

nature. If applied to a video sequence the fascicle configuration is estimated fully independently at every image, i.e. it is not a "tracker", meaning it will not accumulate tracking error, as [6], [10] and [11] may be prone to. However this also means it is not guaranteed to produce a smooth, anatomically plausible evolution in fascicle shape.

In an effort to address the limitations of the currently available approaches, we propose a fully automated approach that: i) provides an automatic segmentation of the fascicle region; ii) describes the average shape configuration across the imaged region of fascicles, but does not impose a global transformation constraint; iii) considers the movements of small feature templates within the image, but avoids interpolating fascicle movements from them directly; iv) explains fascicle structures within a particle-based Bayesian estimation framework, making inferences based on the complete history of observations, rather than the current observation in isolation. To achieve this we make the following specific contributions:

- A low-dimensional curvy-fascicle shape space ( $N$ dimensions for $N$ vertices) constructed from an active shape model (ASM) segmentation of the fascicle region (Section II-A), and suitable for exploration with low numbers of particles (Section II-B).

- A simple but effective observation likelihood for assigning particle weightings, based on the agreement between each fascicle shape hypothesis and the current vesselenhanced ultrasound image (Section II-C).

- A method for estimating suitable dynamical models for particle propagation from the local movements of small image patches in training videos (Section II-D).

- A weak Gaussian Process (GP) prior to constrain particle locations in the state space, ensuring a wide range of plausible fascicle shapes, all of which are locally smooth (Section II-E).

- A fully automatic initialisation procedure, capable of identifying fascicle shapes in real images (Section II-F).

- Evaluation on a range of synthetic and real B-mode ultrasound data with validation against motion capture data and comparisons with currently available approaches (Section II-G).

\section{Methods}

In this section we propose a generic method for fascicle tracking in skeletal muscle, defined using ultrasound images of the medial gastrocnemius (MG), a commonly studied muscle at the back of the lower leg which has a pennate fascicle architecture (obliquely orientated fascicles), see Fig. 1. The method should also be applicable to other muscles, with similar fascicle orientations.

\section{A. Defining Fascicle Shape}

To define fascicle characteristics within an ultrasound image, we construct a number of longitudinal dividing lines, or dividers, stretching across the muscle region of interest. A fascicle is then defined by a collection of offsets along each of these dividers, joined by straight line segments. The more dividers that are used, the more accurate this piece-wise linear approximation to a curved fascicle becomes. Automating this representation requires the automatic identification of the fascicle region in each image, typically around $30 \%$ of a $542 \times 409$ image, see Fig. 1 .

At each frame of an ultrasound sequence, we identify the fascicle region by segmenting the deep and superficial aponeuroses using the active shape model (ASM) formulation, ASM*, proposed in [11]. This produces a set of 76 segmentation points for every image with 38 defining each aponeurosis, see yellow lines in Fig. 2a. Straight dividing lines at the top and bottom of the muscle's fascicle region are then calculated by leastsquares fitting to the deep side of the superficial aponeurosis and superficial side of the deep aponeuroses, respectively, see green and red lines in Fig. 2a. A series of $n$ further longitudinal dividing lines (white lines in Fig. 2a), where $n$ is odd, are then found by interpolating a vertical line of $n$ equally spaced points between the extreme distal and proximal points on each original divider, see white circles in Fig. $2 \mathrm{a}$.

Fig. $2 \mathrm{~b}$ shows a fascicle shape at a time $t$ defined by a collection of offsets from the centre of the image, $\boldsymbol{x}_{t}=$ $\left(x_{t}^{1}, \ldots, x_{t}^{N}\right)^{\top}$, along each of the $N=n+2$ dividers (magenta lines) and connected by piece-wise linear segments (cyan lines). The displacement along the central divider (see red square in Fig. 2a) is always fixed at 0 , so that the fascicle remains stationary relative to the centre of the fascicle region. We do not attempt to track the absolute movements of individual fascicles as they translate across the fascicle region during a movement, but instead try to track changes in the average shape of all fascicles that are visible across the fascicle region. The reader may find it helpful to view the tracking results in the supplementary videos for examples of how segmentations, dividers and fascicle configurations change over time. For all experiments reported here $N=7$ dividers.

\section{B. Multiple Hypothesis Fascicle Tracking}

We adopt a Bayesian multiple hypothesis approach to recover the changing fascicle shape, $\boldsymbol{x}_{t}$ (see Section II-A), during an ultrasound video sequence. A particle filter [16] is used to propagate a discrete approximation to the posterior distribution over time $t=0,1, \ldots, T$ given the sequence of ultrasound image observations $\boldsymbol{z}_{0}, \boldsymbol{z}_{1}, \ldots, \boldsymbol{z}_{T}$, 


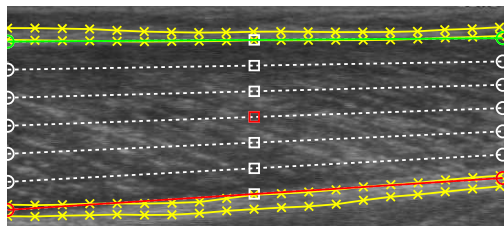

(a)

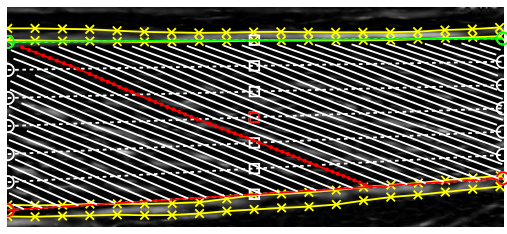

(d)

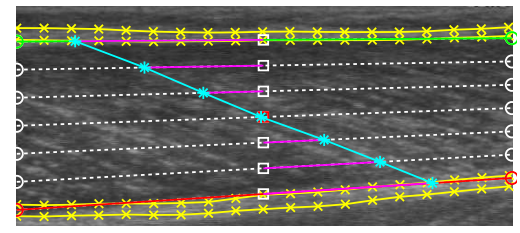

(b)

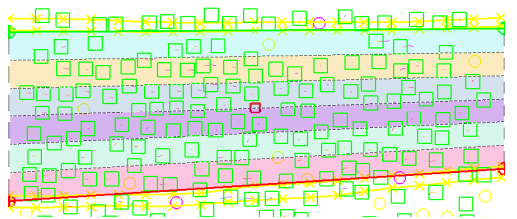

(e)

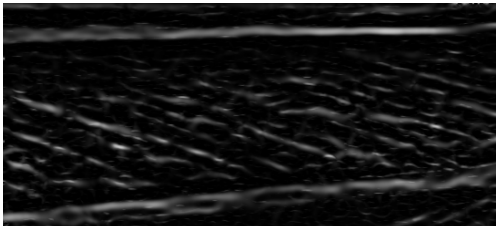

(c)

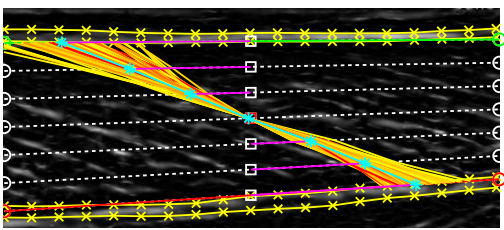

(f)

Fig. 2. B-mode ultrasound images of the gastrocnemius muscle (MG) in the lower leg: (a) ASM* [11] allows for a fully automatic definition of the fascicle region and calculation of $N=7$ longitudinal dividers; (b) A fascicle configuration defined by 7 divider offsets (magenta), connected by straight line segments (cyan); (c) Result of applying a vessel enhancement filter [15] to the B-mode image; (d) A dense fascicle grid derived from the fascicle shape hypothesis, the set of $P_{t, i}^{(q)}=60$ samples for a single fascicle have been highlighted (red dots); (e) KLT tracks are associated with different dividers (coloured segments) for dynamical model and texture estimation (B-mode image removed for clarity); (f) Expected fascicle shape (cyan) superimposed over a set of $Q=100$ hypotheses, coloured proportional to weighting (yellow - 0, red - 1).

$$
\begin{aligned}
p\left(\boldsymbol{x}_{t} \mid \boldsymbol{z}_{0}, \boldsymbol{z}_{1}, \ldots, \boldsymbol{z}_{t}\right)= & \frac{1}{C} \times p\left(\boldsymbol{z}_{t} \mid \boldsymbol{x}_{t}\right) \int_{\boldsymbol{x}_{t-1}} p\left(\boldsymbol{x}_{t} \mid \boldsymbol{x}_{t-1}\right) \ldots \\
& p\left(\boldsymbol{x}_{t-1} \mid \boldsymbol{z}_{0}, \boldsymbol{z}_{1}, \ldots, \boldsymbol{z}_{t-1}\right) d \boldsymbol{x}_{t-1} .
\end{aligned}
$$

This approximation is maintained using a discrete set of $Q=100$ weighted particles,

$$
S=\left\{\left(\boldsymbol{x}_{t}^{(1)}, w_{t}^{(1)}\right), \ldots,\left(\boldsymbol{x}_{t}^{(Q)}, w_{t}^{(Q)}\right)\right\}
$$

where the $q$ th particle $\left(\boldsymbol{x}_{t}^{(q)}, w_{t}^{(q)}\right)$ consists of a unique estimate of the fascicle configuration $\boldsymbol{x}_{t}^{(q)}$ and an associated weight $w_{t}^{(q)}$ approximating the likelihood of the image observation given the fascicle shape, $p\left(\boldsymbol{z}_{t} \mid \boldsymbol{x}_{t}\right)$, see also Section II-C. The particle set must be initialised from an accurate shape configuration $\boldsymbol{x}_{0}$ at time $t_{0}$ (see also Section II-F) and particles are then propagated between images using a dynamical model $p\left(\boldsymbol{x}_{t} \mid \boldsymbol{x}_{t-1}\right)$ that describes our prior belief about how fascicle configurations change over time, see also Section II-D. At any subsequent instant in time we can evaluate the expected fascicle configuration from the particle set using,

$$
E\left[\boldsymbol{x}_{t}\right]=\sum_{i=1}^{Q} w_{t}^{(i)} \times \boldsymbol{x}_{t}^{(i)} .
$$

\section{Observation Likelihood}

For the observation likelihood $p\left(\boldsymbol{z}_{t} \mid \boldsymbol{x}_{t}\right)$ in (1), we consider the relationship of the hypothesised fascicle to a vessel enhanced image, $V$, calculated from the ultrasound image using the approach of Frangi et al. [15]. The result is an image where the value of each pixel represents the maximum vesselness response across a number of different width scales, here $1,2, \ldots, 5$ pixels. A typical result is shown in Fig. 2c.

A fascicle hypothesis $\boldsymbol{x}_{t}^{(q)}$ is used to generate a dense fascicle grid that stretches right across the visible fascicle region. This is done by repeatedly shifting the value of every divider offset by a small, constant value, $\delta_{P}=10$ pixels, to generate a dense grid of neighbouring fascicles. A final grid configuration is shown in Fig. 2d. If the resulting fascicle trajectories coincide with those in the vessel image $V$ then, for the $i$ th fascicle in the grid, we can expect the vesselness responses along that fascicle to be constant. This zero-gradient assumption holds whether the fascicle coincides with an actual fascicle vessel response (bright strand), or with a gap between fascicles (dark strand). The only requirement is that all fascicles in the grid must run parallel with real vessel structures in the image.

At any point in time the $q$ th particle location $\boldsymbol{x}_{t}^{(q)}$ gives rise to a grid with a total of $F_{t}^{(q)}$ fully, or partially visible fascicles. From this grid we extract a dense set of sample points from every fascicle in the grid for comparison with the vesselness map. This is done by interpolating 10 equally spaced co-ordinates along each of the six linear connectors running between dividers, resulting in a set of $P_{t, i}^{(q)} \leq 60$ sample points for the $i$ th fascicle. The red dots in Fig. $2 d$ show an example set for one fully visible fascicle $\left(P_{t, i}^{(q)}=60\right)$. Finally, we calculate the particle's weighting using a sum of squared differences comparison with the vessel image,

$$
w_{t}^{(q)}=\exp \left[-\sum_{i=1}^{F_{t}^{(q)}} \beta_{t, i}^{(q)} / \sum_{i=1}^{F_{t}^{(q)}}\left(P_{t, i}^{(q)}-1\right)\right]
$$

where,

$$
\beta_{t, i}^{(q)}=\sum_{p=2}^{P_{t, i}^{(q)}}\left(V\left(\zeta_{t, i}^{(q)}(p)\right)-V\left(\zeta_{t, i}^{(q)}(p-1)\right)\right)^{2}
$$

and $\left\{\zeta_{t, i}^{(q)}(p)\right\}$ denotes the set of pixel coordinates for the collection of consecutive sample points on the $i$ th fascicle (indexed by $p \in\left[1, \ldots, P_{t, i}^{(q)}\right]$ ). Having calculated all $Q$ particle weights, we normalise to give $\sum_{q=1}^{Q} w_{t}^{(q)}=1$. 


\section{Dynamical Model}

The dynamical model $p\left(\boldsymbol{x}_{t} \mid \boldsymbol{x}_{t-1}\right)$ in (1), is used to move particles through the state space between video frames. It must spread particles widely enough that even the fastest changes in fascicle shape are accounted for. For this purpose we use the addition of Gaussian noise to each particle's location,

$$
\boldsymbol{x}_{t} \sim N\left(\boldsymbol{x}_{t-1}, \boldsymbol{\Sigma}\right) .
$$

The challenge is to estimate suitable values for the variance in each dimension of the state space, which make up the diagonal entries of the matrix $\boldsymbol{\Sigma}$. Furthermore, it must be possible to straightforwardly generate this matrix for different values of $N$, the number of dividers (and the state space dimensionality).

To identify suitable values for the matrix $\boldsymbol{\Sigma}$ for any given ultrasound sequence, we make estimates of the largest shape changes that occur between consecutive images. To do this, we use a KLT tracker to identify [17] and follow [18], [19] distinctively textured image patches in the ultrasound video using the parameters described by [11]. In every image we segment all persisting KLT templates between a set of polygons constructed from the dividers, see coloured segments in Fig. 2e, by associating each square KLT template with the polygon that envelopes its centre-point. We then use the last recovered movement of every template on each polygon to compute a mean drift component along each divider. Having processed all available data we then select each diagonal element of $\Sigma$ as the maximum relative drift observed in the corresponding dimension of the data set.

\section{E. Shape Constraints}

During Bayesian filtering, particles are often exposed to some set of constraints after their propagation, forcing them to lie in a particular subspace of the full state space, e.g. by placing upper and lower limits on each degree of freedom of $\boldsymbol{x}_{t}$ (e.g. [20]) or by learning a latent space with dimensionality reduction (e.g. [21]). These constraints are useful because they stop the inference process generating implausible hypotheses, but they are also inflexible, having been learned from training data in an offline step, and usually only applicable to one particular testing scenario. Here we take a different approach to constraining the state space, basing displacement limits for each divider on the amount of corresponding visual evidence available in the current observation. Large deformations are only permitted where there is sufficient visual evidence to effectively evaluate them via the observation likelihood (Section II-C). This approach, inspired by importance sampling [22], is valuable because in contrast to normal particle filtering [16], it gives consideration to the most recent observation $\left(\boldsymbol{z}_{t}\right)$ during particle propagation. After a particle's configuration has been perturbed using the dynamical model in (6), the resulting collection of divider offsets are revised based on the predictions of a Gaussian Process (GP) shape prior. This revision takes place in order of increasing local image texture; that is - starting with the least textured divider.

GPs are a valuable tool for non-linear regression that can be usefully thought of as providing a probability distribution over functions. Given training data $y_{1}, \ldots, y_{n}$ for a range of input values $x_{1}, \ldots, x_{n}$, the aim is to fit a function to that data and to use it to make predictions about the values of outputs given new inputs. A suitable function can be thought of as a very long (but finite-dimensional) vector where each element contains the value $f(x)$ for a particular value of $x$ [23]. Regression using GPs assumes a Gaussian distribution over all "functions" that explain the training data; that is, the set of outputs relate to the elements of a single vector sampled from an $n$-dimensional Gaussian. The nature of these functions is determined by a covariance function from which we compute the values of the predictive Gaussian's covariance matrix. The form of the covariance function may be tailored to provide results that satisfy prior beliefs about the function.

We use the number of KLT templates associated with each divider as an approximation to the level of visual texture present across that segment of the fascicle region. KLT templates provide a suitable approximation because they capture distinctively textured image patches that are suitable for tracking [17]. In order of increasing texture, each divider offset is shifted (if necessary) to lie within 3 standard deviations of the mean prediction of a GP conditioned on the set of image coordinates, $\mathcal{N}$, of the remaining offsets. We use a GP consisting of a linear mean function, the parameters of which are estimated from the set $\mathcal{N}$, and a radial basis function (RBF) [23] as its covariance function ${ }^{1}$, enforcing a simple and very general prior: that fascicles are locally smooth.

In real ultrasound images we have found that some dividers may, at times, have little or no associated texture, resulting in few (or zero) KLT templates. An analysis across all our video data reveals a lower quartile value of 5 templates per divider. During tracking we exclude any dividers that score below this value on the current image from contributing to the revision of other offsets. This is done by omitting these textureless divider offsets from the set of points upon which the GP's predictions are conditioned, $\mathcal{N}$, giving $|\mathcal{N}| \leq N-1$, and then revising their locations last, conditioned on the newly revised locations of the textured dividers. The effect is to ensure consistent fascicle shape across textureless portions of the image by the interpolation and/or extrapolation of nearby textured divider offsets. The full revision process is illustrated in Fig. 3. Figs. 3b-3f show a series of increasingly minor revisions to offsets along increasingly textured dividers. Finally, Fig. 3 g shows the revision of a divider which is, at the depicted instant, classified as textureless (see black crosses in Fig. 3a). Fig. 2f shows a full particle set that has been propagated using (6), constrained using the GP described above, and weighted using (4).

\section{F. Automatic Initialisation}

Particle filters require initialisation with an accurate system state at the first frame, $\boldsymbol{x}_{0}$. To find this first fascicle shape we use the proposed approach to repeatedly process the first image in a sequence, $z_{0}$. We initialise with a vertical fascicle: all

\footnotetext{
${ }^{1}$ The RBF kernel has two hyperparameters: a signal length scale $\gamma=82$ pixels and signal magnitude $\alpha=28$ pixels. We learn both these parameters from training data, manually clicking clearly visible points along a single good example of a fascicle to give a set of training data $\{f(x)\}$ and then minimising the negative log marginal likelihood with respect to $\gamma$ and $\alpha$.
} 


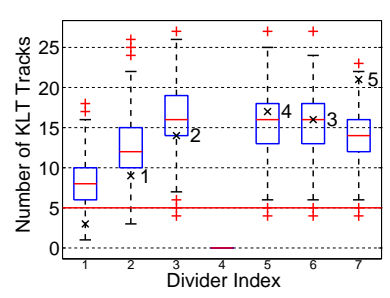

(a)

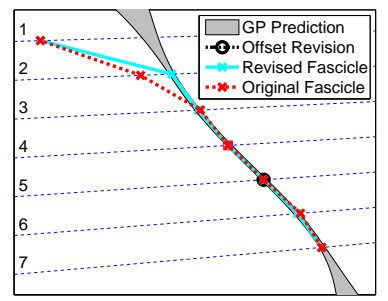

(e)

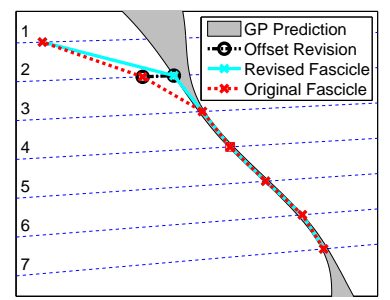

(b)

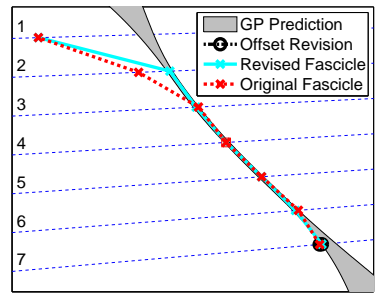

(f)

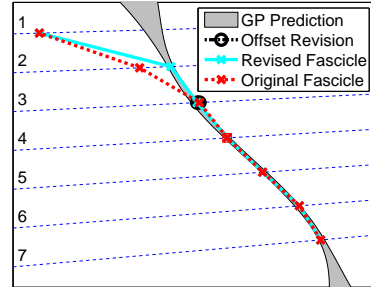

(c)

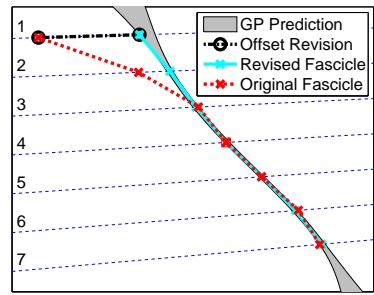

(g)

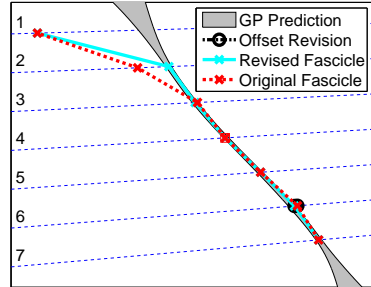

(d)

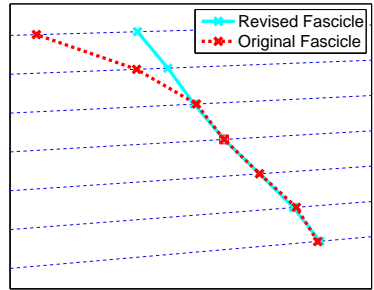

(h)

Fig. 3. Example of Gaussian Process (GP) shape constraints being applied to a single particle after its dispersion by the dynamical model $p\left(\boldsymbol{x}_{t} \mid \boldsymbol{x}_{t-1}\right)$ : (a) Box and whisker plot showing the texture statistics for each divider across a 1000-frame cycling sequence with the minimum texture threshold, dividing textured and textureless dividers, depicted by a solid red line. The individual texture scores for the current image $\boldsymbol{z}_{t}$ are highlighted by black crosses. Textured dividers are shown with numeric labels giving their rank (low-to-high texture) and the order in which they are revised; (b-f) Original offsets (red crosses) are constrained (cyan crosses) to lie within three standard deviations of the mean prediction of a GP conditioned on the remaining textured divider offsets (gray shading); (g) Finally, textureless dividers are revised conditioned on the newly revised textured divider offsets; (h) Original (red) and revised fascicle (cyan).

offsets equal to zero $(\boldsymbol{x}=\mathbf{0})$, and apply 80 tracking iterations to the same image. With a sufficiently large noise matrix, $\boldsymbol{\Sigma}$, our tracker is able to get from this poor initialisation to a shape solution that agrees well with the image, and that our synthetic experiments suggest has an error of just a few pixels per divider (Section III-A). The noise matrix entries were set to half the maximum delta values found across our entire ultrasound dataset (Section III-B). To allow convergence on a single, accurate solution, we gradually reduce the magnitude of particle propagations over time by halving the entries of $\boldsymbol{\Sigma}$ every 20 frames, in an annealing-inspired strategy [24].

\section{G. Alternative Techniques and Parameters}

We compare the performance of the proposed algorithm with two other approaches that are capable of tracking fascicle characteristics. The first is that of Cronin et al. [6] where we make the extensions suggested in their final discussion by defining fascicles using a collection of $N=7$ vertices running across the fascicle region (rather than $N=2$ vertices for straight line fascicles originally used). We also automate the provision of the fascicle region segmentation, using the $\mathrm{ASM}^{*}$ result (rather than a manual first frame procedure as in the original work) enabling us to update the precise region where the approach attempts to estimate the affine warp at every frame. The second is that of Darby et al. [11] where we use $N=7$ probes to capture fascicle characteristics.

\section{Data Collection and Evaluation}

\section{A. Assessment using Synthetic Ultrasound Images}

To allow for quantitative evaluation and cross-comparison between approaches, we generated a number of synthetic Bmode videos showing challenging known curvature conditions.
TABLE I

FASCICLE APPEARANCE: GAUSSIAN DISTRIBUTION PARAMETERS.

\begin{tabular}{|c||c|c|c|c|}
\hline Segment & $\mu$ & $\sigma$ & $\min$ & $\max$ \\
\hline \hline Short (\% of fascicle) & 0 & 8 & 0 & 100 \\
\hline Long (\% of fascicle) & 20 & 8 & 0 & 100 \\
\hline Low Echogenicity (grayscale) & 20 & 11 & 20 & 255 \\
\hline High Echogenicity (grayscale) & 255 & 43 & 20 & 255 \\
\hline
\end{tabular}

B-mode image synthesis [25], [26] works by convolving a noisy model of acoustic impedance (echogenicity) with the point spread function of a theoretical imaging system to produce realistic ultrasound images. Just as with the output of real ultrasound machines, resulting images feature multiplicative noise and - in contrast to previous fascicle simulations (e.g. [12]) - are log-compressed.

To make use of these techniques we must supply information about the echogenicity of the structures within the image, and a small number of constant parameters used in the calculation of the point spread function ${ }^{2}$. The simplest approach is to characterise fascicles with alternating dark (low echogenicity) and bright (high echogenicity) bands before adding noise, e.g. [4], [12]. Here we attempt to increase the challenge provided by such data by additionally considering changes in echogenicity along each individual fascicle.

To synthesise echogenicity maps we take a series of 750 consecutive $\mathrm{ASM}^{*}$ segmentations [11] from images of a real subject's lower leg, and use them to compute the set of dividers (as described in Section II-A) at each instant. We then generate

${ }^{2}$ We use the settings: centre frequency of ultrasound wave $f_{0}=7.5 \times$
$10^{6} \mathrm{~Hz}$; velocity of sound in tissue $c=1540 \mathrm{~ms}^{-1}$; pulse width of wave
$\sigma_{x}=0.5 \mathrm{~m}$; beam width of wave $\sigma_{y}=0.5 \mathrm{~m}$; speckle variance $\sigma^{2}=0.01$. 


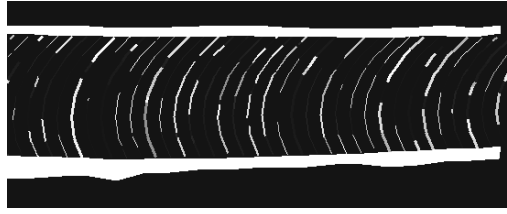

(a)

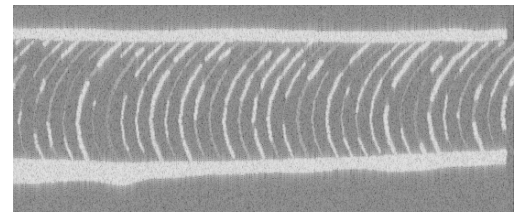

(b)

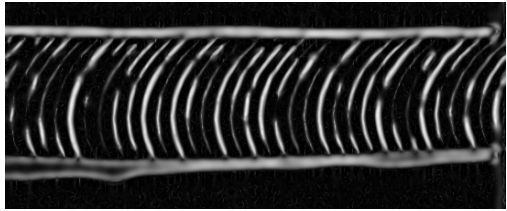

(c)

Fig. 4. Synthetic B-mode ultrasound data for quadratic fascicle shape deformations: (a) Echogenicity map used to generate a single image; (b) Resulting synthetic B-mode image; (c) Resulting vessel enhancement image [15]. The synthetic image generates a similar number of "good" tracking features to the numbers reported by [11] for real images of the MG and produces more realistic "broken" vessel responses (see also Fig. 2c for comparison with real data).

a simple "driving" polynomial function (linear, quadratic and cubic are tested), the coefficients of which change gradually over time, but that always passes through the centre-point of the central divider. By calculating the points of intersection between this function and the remaining dividers, we get a changing ground truth fascicle shape from which we generate dense fascicle grids as described in Section II-C with $\delta_{P}=$ 15 pixels. By drawing out every fascicle in this grid (with a randomly chosen width of between 1 and 4 pixels) to a single image, we generate a single echogenicity map.

To generate echogenicity textures for individual fascicles in the grid we sample from two simple empirical models of fascicle echogenicity. We have observed that the vessel structures visible in the fascicle regions of real B-mode images can be broadly divided between two groups: predominantly light-coloured strands, interrupted by dark-coloured segments; and predominantly dark-coloured strands, interrupted by lightcoloured segments. To represent these two classes in our synthetic echogenicity maps, we draw each fascicle as a collection of individual segments, the length and impedance of which are sampled from the range-limited Gaussian distributions given in Table I. A final result for the quadratic driving function is shown in Fig. 4a. These echogenicity maps are then convolved with the point spread function to create synthetic ultrasound images (Fig. 4b). In each synthetic tracking scenario both baselines (Section II-G) were initialised with the ground truth fascicle shape at $t_{0}$, while the proposed scheme was initialised using the automatic procedure described in Section II-F. Accuracy scores for this automatic procedure are shown in Table II. The noise matrix $\boldsymbol{\Sigma}$ (see Section II-D) was estimated from the synthetic sequence being tracked.

In contrast to the two baselines the proposed approach is designed to estimate average fascicle shape changes regardless of global fascicle movements. I.e. it should produce the same result even if fascicles move at the same time as undergoing shape changes. To test this claim we apply the proposed approach to a second synthetic condition in each of the three scenarios (linear, quadratic, cubic) where fascicles additionally undergo longitudinal translations (at a rate of $25 \mathrm{pix} / \mathrm{sec}$ ) at the same time as their shape deformations. We encourage the reader to view this data in the supplementary videos.

TABLE II

INITIALISATION ERRORS: SYNTHETIC DATA (PIXELS/DIVIDER),

\begin{tabular}{|c|c|c|}
\hline Linear & Quadratic & Cubic \\
\hline \hline 3.50 & 2.52 & 5.25 \\
\hline
\end{tabular}

\section{B. Assessment using Real Ultrasound Images}

Ultrasound image sequences (Aloka ProSound-5000) were collected from $\mathrm{MG}$ on the left leg of 11 participants (10 male, mean \pm SD: Height $182.4 \pm 9.4 \mathrm{~cm}$; Weight: $79.5 \pm 13.8 \mathrm{~kg}$ ) during: i) a series of heel raises, which involved rising onto tip toes and back down; ii) walking on the spot and iii) cycling on a stationary cycle ergometer (130W power, cadence: 40 $\mathrm{rpm})$. All participants provided written informed consent and all procedures were approved by the local ethics committee.

The ultrasound probe (linear, $7.5 \mathrm{MHz}, 50 \mathrm{~mm}$ field of view) was placed over the mid-belly region of $\mathrm{MG}$ along the mediolateral mid-line of the muscle, aligned to the fascicle plane. It was secured with a custom made foam holder with Velcro strapping. Kinematics of the foot, lower leg and thigh segments were recorded using motion capture $(100 \mathrm{~Hz}, 10$ camera VICON Motion Capture, Oxford, UK), with markers placed over the toe (2nd metatarsal head); heel (calcaneous at same height as the toe marker); ankle (lateral malleolus); tibia; knee (lateral epicondyle); thigh; pelvis (anterior and posterior superior iliac spines) of both legs. In one participant, activation of MG was recorded using surface electromyography $\left(1000 \mathrm{~Hz}\right.$, Trigno ${ }^{\mathrm{TM}}$ Wireless, Delsys, Boston, USA), with the electrode placed adjacent to the centre of the ultrasound probe. Synchronous data collection was co-ordinated using custom written MATLAB code, generating a common trigger signal to initiate motion capture and ultrasound image capture $(25 \mathrm{~Hz}$, via DT3120 PCI frame grabber, Data Translations, Germany).

In order to focus on the accuracy of fascicle tracking, we processed these sequences as known subjects. This meant we included a sequence of specifically recorded images from the test subject (performing a different action) in the training set for $\mathrm{ASM}^{*}$ in order to find good segmentations, and we also estimated the noise matrix $\boldsymbol{\Sigma}$ (see Section II-D) from the same sequence being tracked. For those interested in an unknown subject scenario, [11] gives details on segmentation techniques, and a sufficiently large noise matrix can be found by processing sequences from other subjects, although it may require more particles if very large. The proposed approach and both baselines were initialised with the same fascicle shape, found with the automatic procedure in Section II-F.

\section{Fascicle Shape Evaluation}

Tracking using both the proposed approach and the existing alternatives [11], [6] generates a relatively sparse set of $N=$ 7 points; see for example the blue asterisks in Fig. 5a. To evaluate and compare the different approaches we use these 


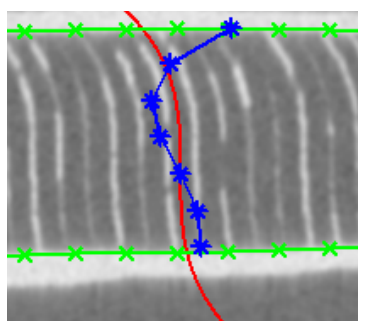

(a)

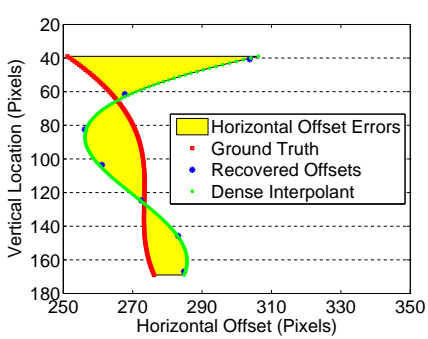

(b)

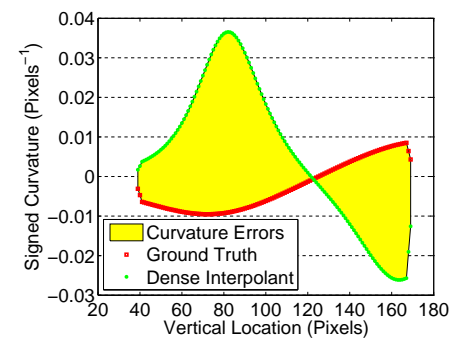

(c)

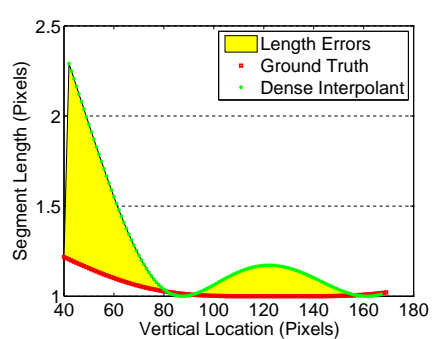

(d)

Fig. 5. Synthetic data evaluation: (a) a poor tracking result (selected for clarity of comparison) with ground truth fascicle shape (red) and tracked fascicle (blue); (b) horizontal offset errors between the true and tracked fascicle; (c) signed curvature errors between the true and tracked fascicle; (d) segment length errors between the true and tracked fascicle.

points to calculate a dense interpolant from which we can compute measures such as local curvature (see below) at every vertical pixel coordinate on the fascicle region. The interpolant is taken as the mean prediction of the GP defined in Section II-E, conditioned on the $N=7$ tracking points (see green circles and blue asterisks in Fig. 5b). The use of a GP avoids the need to make any assumptions about the nature of the fascicle trajectory (e.g. that it is a polynomial [9]) beyond that it is locally smooth.

1) Synthetic Data: In the case of the synthetic ultrasound data we also know the ground truth fascicle shape; see for example the red line in Fig. 5a. This allows us to compute a number of quantitative error measures between the ground truth and the dense interpolant (red and green in Fig. 5b, respectively). First, we calculate absolute horizontal offset errors between the true and tracked fascicle, yellow region in Fig. 5b. These are the absolute horizontal distances in pixels between the true and tracked fascicle at all vertical pixel locations. Second, we calculate local curvature errors, yellow region in Fig. 5c. Following Stark and Schilling [9] we calculate local fascicle curvature measures using the FrenetSerret formula. For a curve defined by a collection of $x$ and $y$ coordinates, $\left\{x_{1}, x_{2}, \ldots, x_{P}\right\}$ and $\left\{y_{1}, y_{2}, \ldots, y_{P}\right\}$, the curvature at the $p$ th point is given by

$$
\kappa_{p}=\frac{\left|x_{p}^{\prime} y_{p}^{\prime \prime}-y_{p}^{\prime} x_{p}^{\prime \prime}\right|}{\left(x_{p}^{\prime 2}+y_{p}^{\prime 2}\right)^{3 / 2}}
$$

where the primes denote local first and second order derivatives. We also multiply by the sign of the second derivative to give a signed local curvature [12]. Finally, we calculate the total length error between the true and tracked fascicle, yellow region in Fig. 5c.

2) Real Data: For real ultrasound data there is no ground truth fascicle with which to compare. Instead we must extract measures relating to the tracked fascicle shape for comparison against the synchronised laboratory measurements described in Section III-B. Following [6] we calculate the total length of the dense interpolant and following [12] we calculate the RMS of all local curvatures as a single scalar measure of "curvyness", $\kappa_{R M S}=\sqrt{\sum_{p=1}^{P} \kappa_{p}^{2}}$. Whereas for synthetic data we report local curvature errors in units of pixels ${ }^{-1}$, we report RMS curvatures for real data in units of $\mathrm{m}^{-1}$ to allow easy comparison with previous studies. One pixel in our real ultrasound images is equal to $0.155 \mathrm{~mm}$.

\section{EXPERIMENTAL RESULTS}

\section{A. Analysis of Synthetic Data}

Average errors for synthetic fascicle shape changes are given in Table III. The proposed approach gives consistently low horizontal offset errors for all three shape conditions (linear, quadratic and cubic), deforming to give an accurate approximation to the ground truth fascicle at all times. This remains the case when fascicles are allowed to translate in addition to changing shape, see bracketed scores in the "proposed" columns. The approach of Cronin et al. [6] outperforms the proposed approach for the linear case, producing lower horizontal offset and length errors, and very low local curvature errors. For the quadratic and cubic cases, however, the changing fascicle shape is not tracked and the initialising fascicle remains almost stationary. The approach of Darby et al. [11] gives reasonable approximations to fascicle shape in the two non-linear cases (e.g. lower local curvature errors for the cubic case), but individual probes tend to drift over time causing high fascicle length errors. The supplementary videos show tracking results for all three algorithms on all conditions.

\section{B. Analysis of Real Data}

An example of mean fascicle lengths and RMS curvatures predicted in one representative participant by each of the tested approaches is shown in Fig. 6. Fascicle lengths predicted by the proposed approach oscillate around $60 \mathrm{~mm}$ as heel height changes. In contrast, length changes predicted by the other approaches [11], [6] do not vary greatly within each movement cycle, but do vary between conditions. This is most evident in the results of the Cronin et al. [6] approach, where lengths during walking-on-the-spot are $\sim 40 \mathrm{~mm}$, while during cycling they are nearly three times longer $(\sim 120 \mathrm{~mm})$. The results for curvature show variation in values across movement cycles predicted by the proposed approach. In contrast, curvatures predicted by the approach of Darby et al. [11] are not physiologically realistic, while those predicted using [6] show large curvature occurring during walking-on-the-spot and very minimal values during cycling and heel raises.

Mean fascicle lengths and RMS curvatures, calculated using the proposed approach in one participant are shown in 
TABLE III

SYNTHETIC DATA PERFORMANCE: THREE SIGNIFICANT FIGURES, BEST SCORES IN BOLD.

\begin{tabular}{|l||l|l|l||l|l|l||l||l|l|}
\hline \multicolumn{1}{|c||}{} & \multicolumn{2}{l||}{ Horizontal Offset Errors (pix) } & \multicolumn{2}{c||}{ Length Errors (pix) } & \multicolumn{3}{c|}{ Local Curvature Errors $\left(\mathrm{pix}^{-1}\right)$} \\
\cline { 2 - 10 } & Proposed & {$[11]$} & {$[6]$} & Proposed & {$[11]$} & {$[6]$} & Proposed & {$[11]$} & {$[6]$} \\
\hline \hline Linear & $1.60(1.74)$ & 9.31 & $\mathbf{1 . 2 7}$ & $2.41(2.82)$ & 18.5 & $\mathbf{1 . 4 5}$ & $0.000777(0.000636)$ & 0.0116 & $\mathbf{0 . 0 0 0 3 2 8}$ \\
\hline Quadratic & $\mathbf{1 . 3 3}(1.24)$ & 5.28 & 6.77 & $\mathbf{1 . 8 5}(1.79)$ & 5.89 & 10.1 & $\mathbf{0 . 0 0 1 5 3}(0.00140)$ & 0.0108 & 0.00742 \\
\hline Cubic & $\mathbf{1 . 9 7}(2.13)$ & 9.02 & 8.52 & $\mathbf{5 . 9 4}(6.55)$ & 25.2 & 16.9 & $\mathbf{0 . 0 0 1 7 0}(0.00183)$ & 0.0108 & 0.0134 \\
\hline \hline Average & $\mathbf{1 . 6 3}(1.70)$ & 7.87 & 5.52 & $\mathbf{3 . 4 0}(3.72)$ & 16.5 & 9.50 & $\mathbf{0 . 0 0 1 3 4}(0.00129)$ & 0.0110 & 0.00703 \\
\hline
\end{tabular}
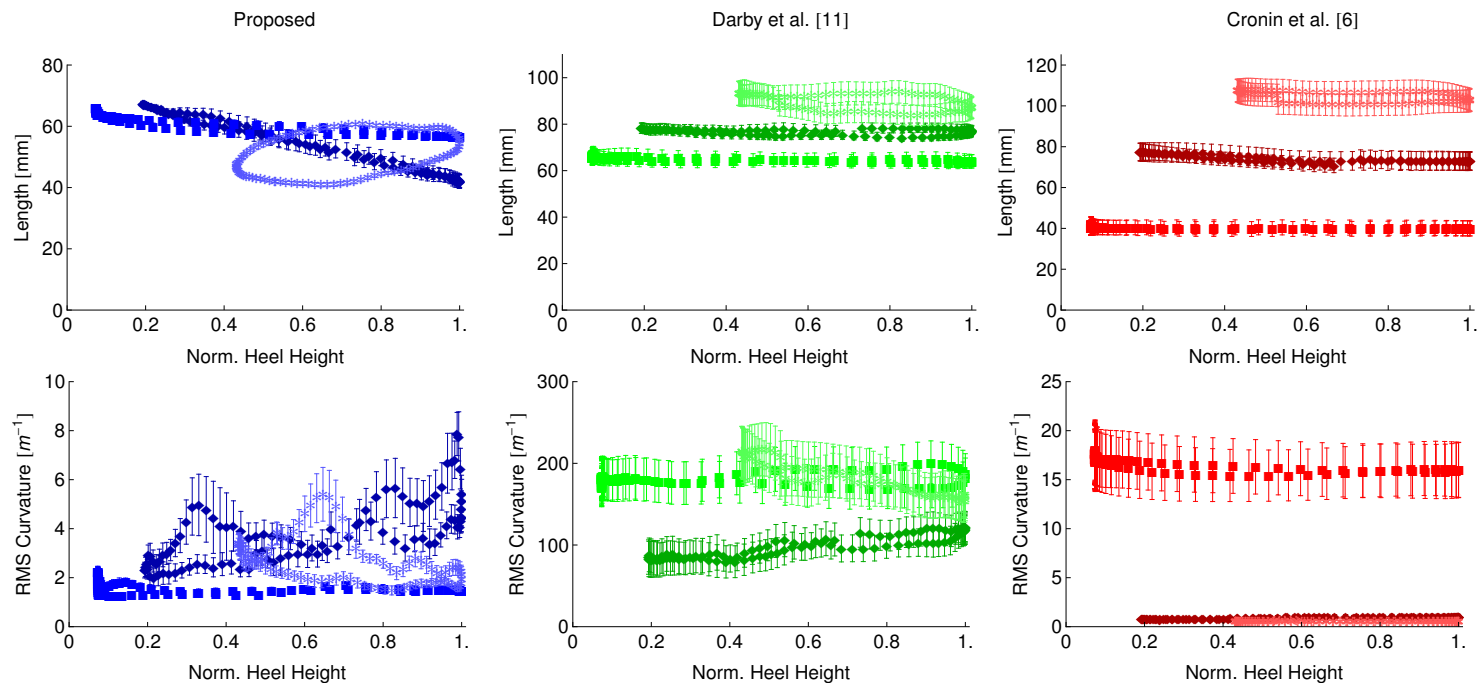

Fig. 6. Example fascicle length (top panel) and RMS curvature (bottom panel) results for each movement task within one representative participant, predicted by: Proposed approach (left column); Darby et al. [11] (central column); Cronin et al. [6] (right column). In each graph movements are distinguished by different hue/shape combinations: Heel raise (dark, diamonds); walking on the spot (mid-colour, squares); cycling (light, stars)

Fig. 7. Again clear, cyclical patterns of length change are apparent. These data include measures of muscle activation and show that during heel raise and cycling activity there is a clear relationship between peak activation, peak curvature and minimum fascicle length. Walking-on-the-spot had the lowest level of activation and the smallest curvatures. Mean minimum and maximum length and RMS curvature values predicted across the sample population are shown in Fig. 8. One-way ANOVA (performed on log transformed data in cases where conditions of equal variance were not met) revealed both length and curvature values predicted by the proposed approach differed significantly within each task (length: $p \leq$ 0.005 ; curvature: $p<0.001)$. Curvature values predicted using [11] also differed significantly within tasks $(p \leq 0.017)$ but were unrealistically high. There were no significant differences in the minimum and maximum curvatures predicted using [6] ( $p \geq 0.159)$ or in the minimum and maximum lengths predicted using [11] ( $p \geq 0.092)$ or [6] ( $p \geq 0.134)$. Coefficient of variation values, calculated from data shown in Fig. 8 , were lower for length values predicted by the proposed approach (mean: $21.52 \%$ range: $19.59-26.14 \%$ ) compared to [11] (mean: $42.46 \%$ range: $28.80-57.29 \%$ ) or [6] (mean: $38.30 \%$ range: $30.07-50.30 \%)$. Much larger variations occurred in the curvature data, with the proposed approach producing the lowest values (mean: $61.20 \%$ range: $40.80-80.22 \%$ ) compared to [11] (mean: $54.89 \%$ range: $37.29-92.88 \%$ ) and [6] (mean:
130.69\% range: $89.18-178.52 \%)$. The supplementary videos show example tracking results for each activity.

\section{DISCUSSION}

\section{A. Performance of the Assessed Approaches}

Results on synthetic data show that for straight fascicle shapes the proposed approach performed well, but that of Cronin et al. [6] was marginally better. However, when fascicle shape deformations become non-linear the proposed approach is able to maintain good tracking accuracy where the alternatives cannot, see Table III and supplementary videos. The assumption of [6], that all fascicle deformations can be described through a series of global transformations between consecutive image pairs, places a restriction on the nature of deformations that can be tracked: it must be possible to reformulate deformations as global affine warps of the entire fascicle region. Where this assumption holds true, e.g. see the linear case in Table III, the restriction usefully constrains changes in fascicle shape and the approach performs well (any affine warp of a straight line is also a straight line). Where this assumption doesn't hold, e.g. quadratic and cubic examples in Table III, the approach is unable to describe the observed shape changes and tracking fails. The contrasting assumption of [11], that deformations in the fascicle region can be safely approximated through the collective movements of local feature patches between consecutive images, imposes 

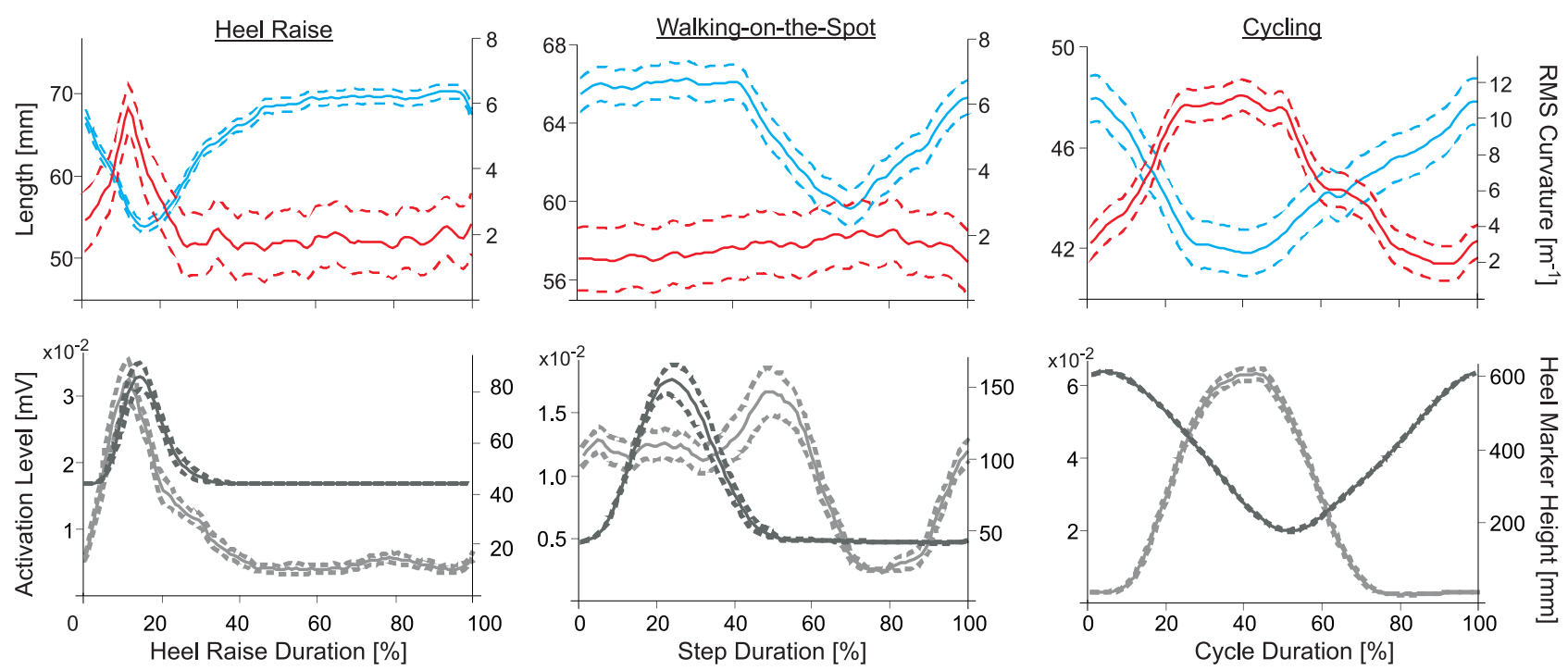

RMS Curvature

Length

Activation Level

Marker Height

Fig. 7. Results from assessment of: heel raises (left column), walking-on-the-spot (middle column) and cycling (right column) in one participant. Top panel: Mean \pm SEM fascicle lengths and RMS curvatures predicted from the proposed approach; Bottom Panel: Mean \pm SEM activation and marker height measures. Mean and SEM values were calculated from data partitioned into individual movement cycles, based on heel marker position, and normalised to 100 percent cycle duration. Solid(broken) lines represent mean(SEM) values. Activation values are the average rectified value of the raw signal, calculated using a $0.25 \mathrm{~ms}$ time window. The cycling sequence is available in the supplementary videos.

no restrictions on possible deformations. However, without any overarching shape constraints this approach is unable to protect against the disturbance of individual fascicle vertices by isolated errors in patch movement estimation, leading to kinks in the tracked fascicle and high length/curvature errors.

Even small errors in the estimation of frame-to-frame shape changes by the baseline algorithms [11], [6] are problematic because they are accumulated, causing tracking to degrade over time. In contrast, the proposed approach attempts to account for the visible fascicle structure in every individual image, allowing it to recover from small tracking errors. This difference is well illustrated by the ability of the proposed approach to initialise itself from a (very poor) vertical fascicle configuration, given only a single image, see Table II and supplementary video. Both [11] and [6] will remain precisely stationary in the same scenario as they have no mechanism to compare fascicle shape against image structure and adjust accordingly. Further, the search for suitable fascicle shapes is made within a Bayesian framework and conditioned on all previous images, not only the latest. This mitigates the effects of single noisy observations and produces a smooth, plausible evolution in fascicle shape. This is in contrast to discriminative trackers (e.g. [12]) which describe single images in isolation and often produce a "jerky" tracking result without any dynamical model to prevent large jumps across the state space.

Analysis of the real ultrasound data further highlighted the influence of the underlying assumptions in each approach. The proposed method, requiring only that fascicles be locally smooth, was able to consistently identify clear cyclical length and curvature changes (Figs. 6 and 7) which are in agreement with previously reported values (e.g. [2], [27]). In addition, consistent and significant differences in minimum and maximum values in each condition across the group of participants were identified, but the same was not apparent for the other approaches assessed. The unconstrained shape predictions resulting from [11] allowed isolated probes to drift, leading to unrealistic predictions of curvature much higher than has been reported in the literature $\left(5-16 \mathrm{~m}^{-1},[12],[2]\right)$. Predicted fascicle lengths did not reflect cyclical changes which would occur in all movements studied (Figs. 6 and 7) and had a tendency to be longer than has been reported in the literature. In contrast, the methods described by Cronin et al. [6], constrained to describe movements through a single global transformation, tended to lose track before becoming "stuck" in an incorrect configuration. This is shown in Fig. 6, where very different fascicle lengths were predicted for each of the conditions studied and changes across the movement cycle were not captured and is also reflected in the high coefficient of variation values found across the data set (see Fig. 8). This failure may be due, at least in part, to nonaffine shape changes in the real data, e.g. where fascicles repeatedly move between straight and curved configurations (see supplementary videos). The original implementation and description of [6] enables manual adjustment of individual tracking points, allowing the operator to correct any perceived errors. Cronin et al. [6] reported this occurred in 6\% of trials studied and more commonly during higher speed movements. However, to capture fascicle shape it is necessary to include $N>2$ vertices, increasing the potential number of points to be adjusted and significantly increasing demands placed on the experimenter and the potential for introduction of bias.

\section{B. Implications for in vivo assessment of fascicle shape}

The proposed methodology for fascicle tracking has two clear advantages over currently available techniques: i) the approach is fully automated; ii) there is no strong assumption 

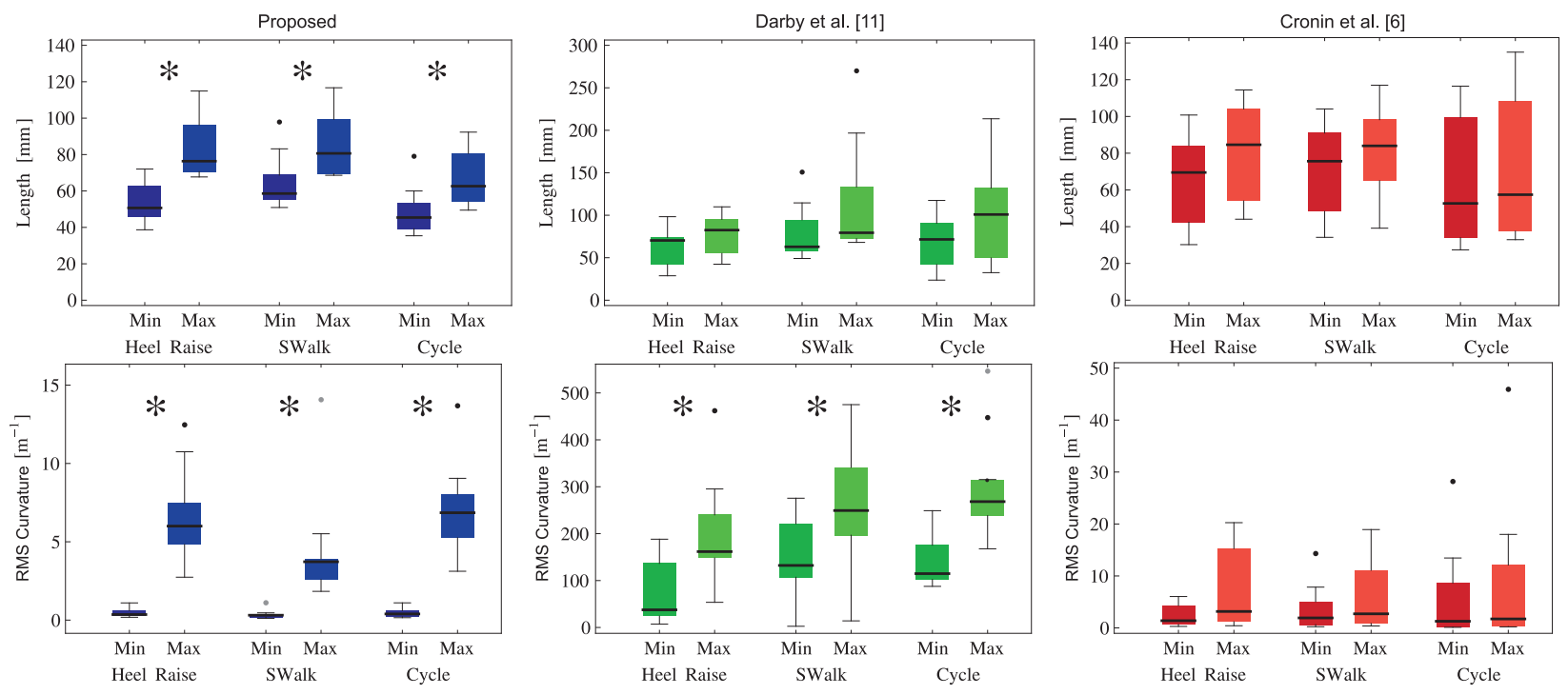

Fig. 8. Box and whisker plots of minimum and maximum (top) length and (bottom) RMS curvature values for the three motor tasks, predicted by: Proposed approach (left column); Darby et al. [11] (middle column); Cronin et al. [6] (right column). Values were calculated from length and curvature measures partitioned into individual movement cycles on the basis of heel marker movement. In all plots the bold central bar represents the median value, bottom and top of the box represent 25th and 75th percentiles respectively and whiskers represent the minimum and maximum values (less outliers, shown as individual points). * indicates significant differences $(p<0.05)$ between minimum and maximum values, determined using One-way ANOVA, $N=11$.

on fascicle shape. Implementing automated image segmentation to identify the fascicle region and automated initialisation of fascicle characteristics provides a significant opportunity to improve the size and robustness of studies of in vivo fascicle dynamics by reducing operator time requirements and potential for the introduction of bias. More significantly however, the ability to study both fascicle length and curvature in an unbiased manner is a significant step forward in enabling fuller investigation of dynamic changes in fascicle geometry which occur to meet different mechanical demands. Differences between, and if different regions of a muscle are scanned, within muscles can now be investigated and a greater level of detail resolved through the facility to quantify local fascicle shape. Such work will have important implications for understanding the functional significance of muscle structure, intramuscular pressure distribution and force transmission.

For robust application of the proposed approach it must be noted that the predicted fascicle characteristics are a representation of the mean values within the fascicle region of interest. As such, tracking will be less robust in situations where fascicle characteristics are highly variable within the field of view or where the movement studied will lead to translation of significantly differently shaped fascicles into the field of view. In addition, the characterisation of a mean fascicle means the proposed approach would not be suitable for studying individual fascicles or small localised or region specific movements for which there are already established approaches [11], [10]. As such it is still important to select the appropriate method of image analysis to address the experimental question at hand.

\section{Conclusions}

The proposed method aims to overcome the weaknesses of existing fascicle tracking approaches, reviewed in Section I, whilst retaining their strengths. Our work has resulted in a flexible fascicle tracker able to describe a very wide range of deformations (e.g. that may simultaneously include strong negative and positive local curvature, as reported in [12]) that need not be describable in terms of global transformations [6]. By making the search for such solutions within a Bayesian framework we also benefit from a smoothly evolving fascicle shape that is conditioned on the entire history of observations, rather than being susceptible to drift from isolated errors in movement estimation [11], or the potential jerkiness of isolated estimation at each frame [12]. The work therefore provides a novel tool with which the functional significance of skeletal muscle geometric properties, across a range of dynamic motor tasks, may be explored in vivo. Processing times are currently between 6-8 seconds per frame (in MATLAB), but the approach is sequential making real time implementation for biofeedback experiments a future possibility.

\section{ACKNOWLEDGMENT}

The authors would like to thank the Wellcome Trust for support of EHT (WT085599MA) and the Dalton Research Institute for support of JD. The function $\mathrm{f}$ cnPseudoBmodeUltrasoundSimulator.m written by Debdoot Sheet and available via the MATLAB File Exchange was used for B-mode ultrasound image simulation.

\section{REFERENCES}

[1] Y. Kawakami, Y. Ichinose, and T. Fukunaga, "Architectural and functional features of human triceps surae muscles during contraction," Journal of Applied Physiology, vol. 85, no. 2, pp. 398-404, 1998.

[2] T. Muramatsu, T. Muraoka, Y. Kawakami, A. Shibayama, and T. Fukunaga, "In vivo determination of fascicle curvature in contracting human skeletal muscles," Journal of Applied Physiology, vol. 92, no. 1, pp. 129-134, 2002. 
[3] T. Miyoshi, T. Kihara, H. Koyama, S.-I. Yamamoto, and T. Komeda, "Automatic detection method of muscle fiber movement as revealed by ultrasound images." Medical Engineering and Physics, vol. 31, no. 5, pp. 558-64, 2009.

[4] M. Rana, G. Hamarneh, and J. M. Wakeling, "Automated tracking of muscle fascicle orientation in B-mode ultrasound images," Journal of Biomechanics, vol. 42, no. 13, pp. 2068 - 2073, 2009.

[5] H. Zhao and L. Q. Zhang, "Automatic tracking of muscle fascicles in ultrasound images using localized radon transform," IEEE Trans Biomed Eng., vol. 58, no. 7, pp. 2094-2101, 2011.

[6] N. J. Cronin, C. P. Carty, R. S. Barrett, and G. Lichtwark, "Automatic tracking of medial gastrocnemius fascicle length during human locomotion," Journal of Applied Physiology, vol. 111, no. 5, pp. 1491-1496, 2011.

[7] J. L. van Leeuwen and C. W. Spoor, "Modelling mechanically stable muscle architectures," Philos Trans R Soc Lond B Biol Sci., vol. 336, no. 1277, pp. 275-292, 1992.

[8] H.-K. Wang, Y.-K. Wu, K.-H. Lin, and T.-Y. Shiang, "Noninvasive analysis of fascicle curvature and mechanical hardness in calf muscle during contraction and relaxation," Manual Therapy, vol. 14, no. 3, pp. $264-269,2009$.

[9] H. Stark and N. Schilling, "A novel method of studying fascicle architecture in relaxed and contracted muscles," Journal of Biomechanics, vol. 43, no. 15, pp. 2897 - 2903, 2010.

[10] I. D. Loram, C. N. Maganaris, and M. Lakie, "Use of ultrasound to make noninvasive in vivo measurement of continuous changes in human muscle contractile length," Journal of Applied Physiology, vol. 100, no. 4, pp. 1311-1323, 2006.

[11] J. Darby, E. Hodson-Tole, N. Costen, and I. D. Loram, "Automated regional analysis of B-mode ultrasound images of skeletal muscle movement," Journal of Applied Physiology, vol. 112, no. 2, pp. 313-327, 2012.

[12] A. I. L. Namburete, M. Rana, and J. M. Wakeling, "Computational methods for quantifying in vivo muscle fascicle curvature from ultrasound images," Journal of Biomechanics, vol. 44, no. 14, pp. 2538 - 2543, 2011.

[13] H. Jiang, P. C. van Zijl, J. Kim, G. D. Pearlson, and S. Mori, "DtiStudio: resource program for diffusion tensor computation and fiber bundle tracking," Comput Methods Programs Biomed., vol. 81, no. 2, pp. 106116,2006

[14] S. Mori and P. C. van Zijl, "Fiber tracking: principles and strategies - a technical review," NMR in Biomedicine, vol. 15, no. 7-8, pp. 468-480, 2002.

[15] R. F. Frangi, W. J. Nissenm, K. L. Vincken, and M. A. Viergever, "Multiscale vessel enhancement filtering," in MICCAI, 1998, pp. 130 137.

[16] M. Isard and A. Blake, "Condensation - conditional density propagation for visual tracking," IJCV, vol. 29, no. 1, pp. 5-28, 1998.

[17] J. Shi and C. Tomasi, "Good features to track," in CVPR, 1994, pp. 593 -600 .

[18] B. D. Lucas and T. Kanade, "An iterative image registration technique with an application to stereo vision," in IJCAI, 1981, pp. 674-679.

[19] C. Tomasi and T. Kanade, "Shape and motion from image streams: a factorization method - part 3 detection and tracking of point features," Computer Science Department, Pittsburgh, PA, Tech. Rep. CMU-CS91-132, April 1991.

[20] R. Sigal, A. Bălan, and M. J. Black, "HumanEva: Synchronised video and motion capture dataset and baseline algorithm for evaluation of articulated human motion," IJCV, vol. 87, no. 1-2, pp. 4-27, 2010.

[21] H. Sidenbladh, M. J. Black, and D. J. Fleet, "Stochastic tracking of 3D human figures using 2D image motion," in ECCV, 2000, pp. 702-718.

[22] M. Isard and A. Blake, "ICONDENSATION: Unifying low-level and high-level tracking in a stochastic framework," in ECCV, 1998, pp. 893908.

[23] C. E. Rasmussen and C. K. I. Williams, Gaussian processes for machine learning. MIT Press, 2006.

[24] J. Deutscher and I. Reid, "Articulated body motion capture by stochastic search." IJCV, vol. 61, no. 2, pp. 185-205, 2005.

[25] J. C. Bamber and R. J. Dickinson, "Ultrasonic B-scanning: A computer simulation," Physics in Medicine and Biology, vol. 25, no. 3, pp. 463479, 1980.

[26] Y. Yu and S. T. Acton, "Speckle reducing anisotropic diffusion," IEEE Transactions on Image Processing, vol. 11, no. 11, pp. $1260-1270$, 2002.

[27] G. Lichtwark, K. Bougoulias, and A. M. Wilson, "Muscle fascicle and series elastic element length changes along the length of the human gastrocnemius during walking and running," Journal of Biomechanics, vol. 40, no. 1 , pp. 157-164, 2007.

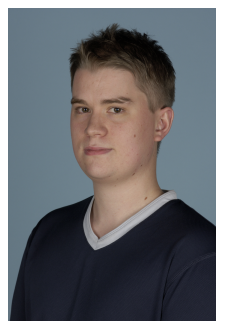

John Darby received a B.Sc. in Computationa Physics from the University of Edinburgh and a Ph.D. degree in computer vision for human pose analysis from Manchester Metropolitan University (MMU). He is currently a Research Associate in the School of Computing, Mathematics and Digital Technology (SCMDT) at MMU, working on visual tracking problems that involve humans.

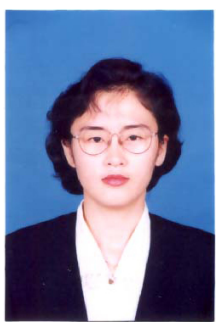

Baihua Li received B.Sc. and M.Sc. degrees in Electronic Engineering from Tianjin University, China and Ph.D. degree in Computer Science from Aberystwyth University, UK. She is a Senior Lecturer in the SCMDT at MMU. Her research experience centres on computer vision, pattern recognition, human motion analysis and behaviour understanding from multi-modality sensor data. She has published over 40 research papers in leading journals and conferences.

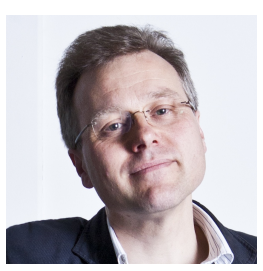

Nicholas Costen received a B.A. in Experimental Psychology from the University of Oxford and Ph.D. degree in Mathematics and Psychology from the University of Aberdeen. He has undertaken research at the Advanced Telecommunications Research Laboratory, Kyoto and at the Division of Imaging Science and Biomedical Engineering, University of Manchester. He is a Reader in Cognitive Computer Vision at MMU where his interests include face recognition and human motion analysis.

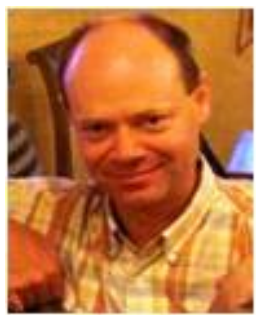

Ian Loram obtained his Ph.D. degree in postural control and applied physiology from The University of Birmingham in 2003. He worked as a Leverhulme Early Career Research Fellow and is currently Professor in Neuromuscular Control of Human Movement at MMU. His work focuses on using biomechanics, control theory, motion analysis and ultrasound/EMG analysis to understand muscle action in the control of posture, movement and human performance.

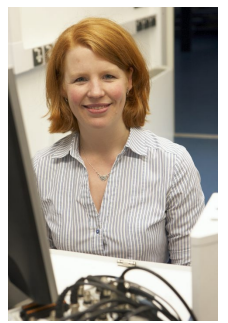

Emma Hodson-Tole obtained her Ph.D. degree in biomechanics and muscle physiology from The University of London in 2007. She worked as a postdoctoral fellow in The School of Applied Physiology at Georgia Institute of Technology, before being awarded a Sir Henry Wellcome Postdoctoral Fellowship and commencing work at MMU. Her work focuses on the integration of neuromuscular activation dynamics, anatomical and physiological characteristics. More details of current projects can be found at: www.irm.mmu.ac.uk. 